\title{
Supressão de ritmo mu durante exposição a estímulos associados a ansiedade social
}

\author{
Suppression of mu rhythm during exposure to social anxiety related stimuli
}

\author{
André Amado Ferreira de Mello', Renato Teodoro Ramos ${ }^{2}$
}

Mello AAF, Ramos RT. Supressão de ritmo mu durante exposição a estímulos associados a ansiedade social / Suppression of mu rhythm during exposure to social anxiety related stimuli. Rev Med (São Paulo). 2017 out.-dez.;96(4):254-63.

RESUMO: O chamado ritmo mu em eletroencefalografia é um padrão de oscilação caracterizado pelo domínio de frequências na banda de $8-13 \mathrm{~Hz}$ e $15-25 \mathrm{~Hz}$. A inibição deste padrão de atividade tem sido associada a atividade de neurônios espelho em áreas motoras e pré-motoras do córtex cerebral. Neurônios espelho, por sua vez, têm sido relacionados à capacidade empática, capacidade esta que pode estar alterada na ansiedade social. O projeto teve como objetivo comparar os padrões de atividade cortical em indivíduos com níveis altos e baixos de ansiedade social durante a observação de uma cena capaz de gerar ansiedade social filmada em duas situações: em terceira pessoa (quando a capacidade empática deve ser evocada) e em primeira pessoa (quando apenas possíveis sentimentos de ameaça a si próprio devem ser evocados). O estudo envolveu 70 voluntários normais selecionados e, a partir da aplicação da escala de Liebowitz para avaliar ansiedade social e da escala EMRI para avaliação da capacidade empática, foram selecionados 16 voluntários, sendo $8 \mathrm{com}$ os maiores escores nestas escalas e $8 \mathrm{com}$ as menores pontuações. A análise inicial do traçado eletroencefalográfico de todos os voluntários não identificou padrões de distribuição de potência de ritmos alpha em regiões fronto-parietais, que poderia sugerir um padrão de atividade associada ao ritmo mu característico de neurônios espelho. No entanto, foi identificada uma diferença significativa na comparação entre padrões de atividade associados a observação dos vídeos em primeira e terceira pessoa $(p=0.007)$. Esta diferença foi também relevante em regiões occipitais, o que poderia estar relacionado à estimulação visual inerente aos estímulos apresentados. Portanto, não foi possível estabelecer uma correlação clara entre os traços de ansiedade social e empatia com uma possível atividade de neurônios espelho (por meio da inibição do ritmo mu). O estudo, porém, evidenciou uma diferença estatística significativa entre os padrões de atividade cortical associados à observação de cenas em primeira e terceira pessoa, independente dos traços ansiosos. Deve-se observar que o estímulo visual utilizado pode ter sido excessivo, induzindo atividades corticais ligadas à atividade perceptiva visual independente do significado ou do contexto que se procurou transmitir ao observador. $\mathrm{O}$ avanço da investigação da hipótese explorada neste projeto demandará novos estudos eventualmente incluindo pacientes com níveis clinicamente relevantes de ansiedade social e a simplificação dos estímulos utilizados.

Descritores: Eletroencefalografia; Neurônios-espelho; Ansiedade; Empatia. ABSTRACT: The so-called mu rhythm in electroencephalography
is an oscillation pattern characterized by the frequency domain
in the band $8-13 \mathrm{~Hz}$ and $15-25 \mathrm{~Hz}$. Inhibition of this pattern of
activity has been associated with the activity of mirror neurons in
motor and premotor areas of the cortex cerebral. Mirror neurons,
in turn, have been related to empathic ability, which capacity may
be altered in social anxiety. The aim of the project was to compare
the patterns of cortical activity in individuals with high and low
levels of social anxiety during the observation of a scene capable
of generating social anxiety filmed in two situations: in the third
person (when empathic capacity should be evoked) and in the
first person (when only possible feelings of self-threat should

1. Faculdade de Medicina FMUSP, Universidade de Sao Paulo, Sao Paulo, SP, BR. Acadêmico de Medicina. Email: andre.amado@ fm.usp.br.

2. Departamento de Psiquiatria, Faculdade de Medicina FMUSP, Universidade de Sao Paulo, Sao Paulo, SP, BR. Orientador, Prof. Dr., graduado em Medicina pela Universidade de São Paulo (USP-1984), doutorado em Psiquiatria pela USP (1995), pós-doutorado no Department of Psychiatry da University of Pittsburgh USA (1996) e no Deparment of Psychology da King's College of London (2005), atualmente Professor Associado. Email: renato.ramos@hc.fm.usp.br.

Endereço para correspondência: André Amado Ferreira de Mello. Rua Barreto Leme, 1887 Ap. 102. Campinas, SP, BR. Email: andre. amado@fm.usp.br 
be evoked). The study involved 70 normal volunteers selected and, from the application of the Liebowitz scale to evaluate social anxiety and the EMRI scale for the evaluation of empathic capacity, 16 volunteers were selected, 8 with the highest scores on these scales and 8 with the lowest scores. Initial analysis of the electroencephalographic tracing of all volunteers did not identify patterns of alpha rhythm power distribution in fronto-parietal regions, which could suggest a pattern of activity associated with the characteristic mu rhythm of mirror neurons. However, a significant difference was found in the comparison between activity patterns associated with observation of first and third person videos $(p=0.007)$. This difference was also relevant in occipital regions, which could be related to the visual stimulation inherent to the presented stimuli. Therefore, it was not possible

\section{INTRODUÇÃO}

Chamado ritmo mu em eletroencefalografia é um padrão de oscilação caracterizado pelo domínio de frequências na banda de $8-13 \mathrm{~Hz}$ e $15-25 \mathrm{~Hz}$. Estas oscilações são limitadas a breves períodos de 0,5 a 2 s de duração e podem ser registradas sobre o córtex sensório-motor de humanos na ausência de movimentos ${ }^{1}$. O ritmo mu, também conhecido como ritmo Rolândico ou sensório-motor, foi descrito na década de 1950, mas recebeu relativamente pouca atenção até recentemente, provavelmente por não ser habitualmente detectado na maioria dos indivíduos ${ }^{2}$. No entanto, técnicas mais sofisticadas, como a análise de componentes independentes têm demonstrado a presença de ritmo mu nos registros eletroencefalográficos da maioria, senão todos os indivíduos adultos saudáveis ${ }^{3}$. Como é observado em momentos de ausência de atividade motora, ou seja, sobre uma área não ativa, o ritmo mu foi inicialmente considerado como reflexo de um estado de repouso similar ao descrito para o ritmo alfa clássico ${ }^{4}$. Este ponto de vista tem sofrido mudanças recentemente a partir de relatos de supressão de ritmos do tipo alfa durante a realização de atividades cognitivas ${ }^{5}$.

A dessincronização do EEG é vista como o resultado da ativação de vias tálamo-corticais e o consequente aumento da atividade cortical, ao passo que sua sincronização se correlaciona com a redução da atividade cortical ${ }^{1}$. Tanto o bloqueio do ritmo alfa com o abrir dos olhos quanto o bloqueio do ritmo mu durante a preparação ou execução de movimentos são exemplos destes mecanismos. O grau de supressão do ritmo mu durante o movimento pode ser expresso a partir da porcentagem de redução do pico de potência em repouso tipicamente da ordem de $61 \%(\mathrm{SD}=25)^{6}$.

Dados experimentais sugerem que as dessincronizações relacionadas a eventos (event-related desynchronization ou ERD) na faixa dos $10 \mathrm{~Hz}$ são um correlato da atividade cortical do processamento de informações, da manutenção da atenção seletiva e do preparo para a atividade motora ${ }^{7}$. to establish a clear correlation between social anxiety traits and empathy with possible mirror neuron activity (through inhibition of mu rhythm). The study, however, showed a significant statistical difference between the patterns of cortical activity associated with the observation of first and third person scenes, regardless of the anxious features. It should be noted that the visual stimulus used may have been excessive, inducing cortical activities linked to visual perceptual activity independent of the meaning or context sought to convey to the observer. The advance of the investigation of the hypothesis explored in this project will require new studies eventually including patients with clinically relevant levels of social anxiety and the simplification of the stimuli used.

Keywords: Electroencephalography; Mirror neurons; Anxiety; Empathy.

Registros de ERD associada ao ritmo mu em adultos começam cerca de dois segundos antes do início do movimento voluntário e sua magnitude parece ser proporcional a complexidade da tarefa motora a ser realizada ${ }^{8}$. A inatividade motora e o nível de atenção parecem ser os principais fatores capazes de afetar a expressão e manutenção do ritmo mu. A indução de um movimento real é um mecanismo robusto de supressão de um ritmo mu pré-existente, mas a atenção por si só é capaz do mesmo efeito, talvez através de mecanismos de comportamentos motores imaginados ${ }^{2}$.

\section{Ritmo Mu e neurônios-espelho}

Neurônio espelho é um neurônio que é ativado quando um animal atua ou observa a mesma ação sendo realizada por outro animal. A ativação destas células, portanto, espelharia o comportamento de outro indivíduo como se o próprio sujeito estivesse realizando esta ação. Descritos por Rizzolatti et al. ${ }^{1}$ a partir de registros de eletrodos em cérebro de macacos, acredita-se atualmente que neurônios espelho existam em diferentes primatas, aves e seres humanos onde sua atividade tem sido descrita em regiões pré-motoras e em córtex parietal inferior.

A correlação entre os ritmos mu e a atividade dos neurônios espelho foi sugerida primeiramente por Altschuler et al. ${ }^{9}$ e tem sido continuamente corroborada desde então. Pineda ${ }^{2}$ propõe que os ritmos mu reflitam a modulação da atividade de neurônios motores por células com função espelho localizadas no córtex pré-motor. Em repouso, neurônios sensório-motores disparam em sincronia levando ao aumento da amplitude de ondas na faixa de 8-13 Hz. A potência desta atividade registrada sobre regiões parietais é reduzida por movimentos autoiniciados, imaginados ou observados ${ }^{10}$. Estudos com ressonância magnética funcional também tem corroborado esta hipótese ${ }^{11}$.

Além de sua responsividade à imaginação de atividades motoras, o sistema de neurônios espelho humano 
parece estar envolvido em atividades cognitivas superiores. Rizzolatti et al. ${ }^{12}$ sugerem que a capacidade de associar representações visuais de uma ação observada com uma ação a ser realizada pelo indivíduo seja fundamental para o aprendizado imitativo ${ }^{12}$.

Diferenças na evocação de ritmos mu entre indivíduos com autismo e controles tem sido relacionadas a dificuldade de aquisição de linguagem, imitação e empatia nesta população ${ }^{2}$. Além disso, existem evidências de que expressões faciais e a percepção do significado destas possam também depender deste tipo de atividade espelho ${ }^{13}$. Stroganova et al. ${ }^{14}$ demonstraram que ritmos do tipo alfa podem ser bloqueados pela estimulação visual mesmo em crianças de 7 a 12 meses de idade, sugerindo uma semelhança funcional com adultos em relação à ocorrência deste ritmo. Estes mesmos autores relataram um aumento expressivo da atividade em 6 a $8 \mathrm{~Hz}$ em regiões parieto-occipitais na ausência de estimulação visual em comparação com uma situação de atenção relaxada. A frequência média deste assim chamado ritmo alfa central evolui a partir do primeiro ano de vida de cerca de $7 \mathrm{~Hz}$ até estabilizar-se por volta de $10 \mathrm{hz}$ na adolescência, sugerindo que esta progressão esteja associada ao aprendizado motor. Estudos recentes sugerem uma equivalência entre o ritmo central em criança e o ritmo mu sensório-motor em adultos ${ }^{14}$.

Em resumo, como proposto por Pineda ${ }^{2}$, a fenomenologia dos ritmos mu apresenta uma alta semelhança com o observado para a atividade neuronal espelho. Ambas são sensíveis ao movimento e à atividade imaginativa e a superposição de seus substratos anatômicos sugere que ambas as atividades sejam fundamentais para o funcionamento cognitivo superior.

\section{Neurônios espelho e ansiedade}

O papel dos neurônios espelho na gênese dos sintomas de ansiedade tem sido estudado em humanos e em animais através, entre outras possibilidades, do modelo conceitual de avaliação de riscos. A avaliação de riscos é um modelo de comportamento envolvido na detecção e análise de ameaças e de situações onde estas ocorrem. Em animais, este é um processo central na escolha das defesas específicas, como fuga, congelamento, ameaça defensiva ou ataque defensivo. Este processo altamente adaptável leva em conta características como o tipo e localização da ameaça e da situação (por exemplo, a presença de uma via de fuga ou esconderijo), combinando-os para prever qual defesa específica é ideal diante dessa combinação particular de ameaça e situação. A avaliação de risco é particularmente associada à ambiguidade do estímulo ou do possível resultado dos comportamentos defensivos disponíveis. Este comportamento é também crucial para determinar em que momento a ameaça não está mais presente, permitindo um retorno ao comportamento defensivo normal. Embora este padrão comportamental tenha sido descrito em detalhe em roedores, presume-se que a ruminação cognitiva possa ser uma forma especificamente humana de avaliação de risco, associada com a ansiedade ${ }^{15}$.

Estas observações são importantes também do ponto de vista clínico. Em modelos animais, comportamentos de avaliação de risco são afetados por medicamentos ansiolíticos, mas respostas de fuga, uma defesa dominante em muitas situações comuns, mostra um perfil de resposta farmacológica mais próxima ao do transtorno de pânico. Avaliações de risco e fuga também parecem mostrar algumas diferenças consistentes em termos de padrões de ativação regionais cerebrais, sugerindo um potencial de diferenciação biológica de ansiedade e sistemas de medo/ pânico. Uma possibilidade especialmente interessante é que os neurônios-espelho possam responder a alguns dos mesmos tipos de diferenças situacionais que são analisados durante a avaliação de risco, sugerindo um papel funcional adicional para esses neurônios ${ }^{15}$.

\section{Ansiedade social}

O foco dessa pesquisa é um dos possíveis tipos de ansiedade conhecida como ansiedade social. A ansiedade social é caracterizada pelo medo de uma ou mais situações nas quais o indivíduo é exposto à possível avaliação por parte dos outros, quando realiza atividades como, por exemplo, comer, beber, falar em público, ser o centro das atenções ou interagir com o sexo oposto. O indivíduo teme fazer algo errado ou comportar-se de maneira humilhante ou embaraçosa. A esquiva destas situações é frequente, porém, quando tais situações são inevitáveis, podem ocorrer manifestações importantes de ansiedade frequentemente acompanhadas de sintomas autonômicos como: rubor, taquicardia e tremores.

Os pacientes com ansiedade social, embora reconheçam a inadequação de seus medos, com relação à racionalidade e com relação ao excesso, não conseguem inibir suas respostas frente ao estímulo.

Como todas as demais formas de manifestação da ansiedade, a ansiedade social também afeta processos cognitivos, perceptuais e de aprendizado. Além disso, a ansiedade social tende a induzir erros e distorções perceptivas de tempo e espaço bem como na atribuição de significados às pessoas e situações.

A ansiedade social parece ser um fenômeno comum, modulado por mecanismos neurobiológicos associados aos sistemas de defesa. A complexidade da interação social humana faz com que estes mecanismos possam também ser ativados por estímulos de natureza simbólica. Perceber as intenções ou impressões de outras pessoas é particularmente importante para que estas estratégias e comportamentos sejam adequadamente usados em favor do indivíduo.

Ter um contato empático com outras pessoas torna- 
se, portanto, fundamental para que as reações de ansiedade social sejam ativadas ou inibidas de forma adequada e propiciem um convívio saudável.

\section{Empatia}

A empatia é definida como a capacidade de compartilhar emoções, bem como a capacidade de entender os pensamentos, desejos e sentimentos do outro. Em psicologia, empatia é descrita como a capacidade de experimentar e compreender o que os outros sentem, sem confusão entre si mesmo e o outro. Em última instância, ela é considerada um mecanismo adaptativo importante, pois propicia respostas como alarmes de grupo, interação mãe-bebê e comportamentos reprodutivos ${ }^{12}$.

A empatia é uma construção psicológica baseada tanto em componentes cognitivos, quanto afetivos, sugerindo a existência de ao menos dois mecanismos empáticos conhecidos como empatia cognitiva e empatia emocional. A empatia cognitiva está relacionada à capacidade de compreender a perspectiva psicológica das outras pessoas ou a capacidade de se engajar no processo cognitivo e adotar o ponto de vista psicológico do outro ${ }^{16,17}$.

Por sua vez, a empatia emocional está relacionada à habilidade de experimentar reações afetivas, por meio da observação da experiência da outra pessoa. Nela estão incluídos o contágio emocional, o reconhecimento da emoção e o compartilhamento desta. A importância desta distinção entre tipos de fenômenos empáticos se justifica pelas evidências de que diferentes estruturas corticais possam estar relacionadas a manifestação de cada uma delas $^{18}$.

Além disso, existem evidências de que neurônios espelho tenham um papel fundamental na vivência e expressão da empatia e anormalidades da capacidade empática tem sido relacionadas com certos aspectos clínicos de condições como esquizofrenia e autismo ${ }^{19,20}$.

\section{Empatia e neurônios espelho}

Existe uma proximidade conceitual entre empatia e funções do tipo das realizadas pelos neurônios espelho. Esta similaridade relaciona-se à ideia de mimetismo onde conteúdos cognitivo-motores das ações humanas são vistos como uma forma de representação necessária tanto para a ação quanto para a percepção do mundo exterior. Imitação e mimetismo são elementos fundamentais dos processos de interação social e de aprendizado e parecem ser em grande parte geneticamente determinados e automáticos, de forma a facilitar a atividade social ${ }^{21}$.

O papel dos neurônios espelho no comportamento empático seria o de permitir uma representação interior de ações, movimentos e, principalmente, de intenções. De fato, parece que a supressão do ritmo mu, em indivíduos normais, é mais intensa quando os mesmos são expostos a figuras de faces expressando aprovação ou recompensa em comparação a faces expressando reprovação, sugerindo que a atividade empática possa modular mecanismos de avaliação de recompensa e ameaças de natureza social ${ }^{5}$.

Comportamentos ligados a noção de moralidade também tem sido descritos como relacionados a atividade deste grupo de neurônios. Por exemplo, áreas do córtex pré-frontal parecem estar envolvidas no acompanhamento dos efeitos do comportamento do próprio indivíduo do valor de suas próprias ações em contextos sociais. Estas observações sugerem que a cognição moral poderia surgir como consequência da atividade das redes de processamento cerebrais emocionais, provavelmente envolvendo neurônios espelho ${ }^{22}$.

Presume-se que a atividade ídeo-motora envolvida nas ações humanas tenha uma forma de representação comum para a ação e percepção que facilita a imitação. Para que este processo seja otimizado, a existência de uma capacidade inata de observação e representação de intenções seria particularmente vantajosa ${ }^{23}$.

Os padrões de apresentação de ritmos mu associados a situações de percepção e interpretação de situações sociais têm sido estudados principalmente através da apresentação de estímulos faciais, mas outros componentes do contexto que gera estas emoções ainda não foram adequadamente estudadas $^{24,25}$.

\section{OBJETIVOS}

Investigar nesta pesquisa as seguintes hipóteses:

1. O contato social é mediado pela capacidade empática do individuo;

2. Indivíduos com níveis maiores de ansiedade social devem exibir diferenças em sua capacidade empática em relação a indivíduos com níveis menores de ansiedade social;

3. Esta diferença na capacidade empática pode se refletir na atividade dos neurônios espelhos e, portanto, poderiam ser identificadas a partir da análise da supressão de ritmo mu em áreas frontoparietais;

4. Para que esta diferença seja detectada, é necessária uma comparação entre os padrões de atividade associados quando a) o indivíduo observa outra pessoa em situação supostamente de ameaça social e b) quando ele mesmo sente-se exposto a este tipo de ameaça;

5. A identificação do envolvimento de neurônios espelho na ansiedade social pode contribuir para o entendimento da fisiopatologia da fobia social, um transtorno mental frequente e incapacitante.

\section{Estratégia experimental}

Para tal investigação, compararam-se os padrões de atividade cortical em indivíduos com níveis altos e baixos 
de ansiedade social (baseado na Escala de Liebowitz) durante a observação de uma cena capaz de gerar ansiedade social filmada em duas situações: em terceira pessoa (quando a capacidade empática deve ser evocada) e em primeira pessoa (quando apenas possíveis sentimentos de ameaça a si próprio devem ser evocados).

\section{MATERIAL E MÉTODOS}

\section{Sujeitos}

Através da aplicação da Escala de Liebowitz ${ }^{26}$ para a Ansiedade Social e da Escala Multidimensional de Reatividade de Davis (EMRI) ${ }^{27}$ num grupo de 70 estudantes universitários, foram selecionados e convidados para participar do estudo 16 indivíduos, com escores variados (inicialmente foram selecionados e convidados os de maior e menor escore) na tabela montada com as duas escalas, usando como referência, para o recrutamento, a Escala de Liebowitz. Após a leitura e assinatura do termo de consentimento, os voluntários participaram da próxima etapa do estudo, a da realização do EEG.

\section{Registro eletroencefalográfico}

O registro do EEG foi feito nas seguintes condições: voluntário sentado em um estado de repouso, com a touca de 128 eletrodos na cabeça de acordo com o sistema internacional de referência 10-5. Eletrodos de eletromiografia para monitorização de movimentos oculares também foram usados. Solicitamos que os indivíduos mantivessem o olhar fixo na tela evitando movimentos oculares (exceto piscadas simples) e outros movimentos faciais ou corporais (como mordida).

Antes do experimento, dois minutos de atividade basal cerebral foram registrados em cada uma das seguintes situações: com luz acesa e indivíduo de olhos abertos; indivíduo com olhos fechados em ambiente com a luz apagada. $\mathrm{O}$ experimento consistiu na apresentação de três vídeos, cada um representando uma condição experimental. Primeiramente, dois vídeos foram apresentados em ordem aleatória: um filmado em primeira pessoa e outro em terceira pessoa. Em seguida, foi mostrado um terceiro vídeo, que consiste na repetição do vídeo em terceira pessoa, porém com a imagem em movimento. Essa última condição serve como um controle, para verificar se eventuais diferenças eletroencefalográficas encontradas entre as duas primeiras condições não seriam atribuídas à maior movimentação da câmera no vídeo em primeira pessoa. Os filmes, de cerca de 120 segundos cada, retratam a mesma situação: uma pessoa passando por situações de embaraço social, ao se dirigir para uma reunião de trabalho.

Os vídeos foram apresentados à uma distância de aproximadamente $60 \mathrm{~cm}$ dos olhos, com área de imagem de cerca de $8 \times 8$ graus visuais. O registro eletroencefalográfico foi realizado com frequência de amostragem de $1000 \mathrm{~Hz}$, com referência biauricular. Ao final do experimento, a duração do EEG foi de um pouco mais de dez minutos.

\section{Metodologia de processamento e análise dos dados}

A baixa relação sinal-ruido e a baixa precisão espacial representam os principais fatores que dificultam a análise dos sinais eletroencefalográficos. Portanto, é preciso um prévio processamento, no qual se procura isolar as atividades cerebrais de interesse. Tanto o processamento como a análise dos sinais eletroencefalográficos foram executados com o programa Matlab v. R2014a (The Mathworks).

Primeiramente, componentes periódicos com frequências inferiores a $2.5 \mathrm{~Hz}$ e superiores a $40 \mathrm{~Hz}$ foram removidos do sinal com o uso de filtros digitais (Butterworth de quarta ordem aplicado em ambos os sentidos para evitar distorções de fase). Em seguida, variações de voltagem provenientes de movimentos oculares (associado a movimentos que não puderam ser evitados durante o experimento, tais como piscadas, sacadas e demais movimentos dos olhos) foram removidas com a utilização de análise de componentes independentes ${ }^{28}$, técnica na qual se procura reconstituir sinais das fontes geradoras do EEG, partindo do pressuposto de que eles são independentes estatisticamente entre si. Sua eficácia para identificação e eliminação de atividades elétricas de origem ocular se dá pelo fato desses artefatos de fato serem independentes em relação aos sinais cerebrais. Os sinais cerebrais que não eram de interesse para o estudo também foram retirados inicialmente com esse método de análise dos componentes independentes.

Os intervalos de registro utilizados para a análise foram os últimos $65536 \mathrm{~ms}$ (1.09 minutos) de cada vídeo. Para cada participante, canal e condição, foi calculado espectro de potência de Fourier com método de Welch (janelamento $1024 \mathrm{~ms}$ )29. Nesse espectro, identificou-se o pico individual de alpha como a frequência de maior potência na banda de 8 a $14 \mathrm{~Hz}$ no eletrodo Cz mesclandose as três condições. A banda alpha utilizada para análise foi determinada como a potência média na banda de 4 $\mathrm{Hz}$ centrada no pico individual de alpha. Para todos os indivíduos, banda beta foi definida como a potência média nas frequências entre 15 e $25 \mathrm{~Hz}$.

Em análise exploratória, foi realizada comparações das potências alpha e beta (separadamente para cada banda) entre as 3 condições com ANOVA de medidas repetidas (uma via, verificando-se efeito da condição) para cada canal. Nas regiões com eventuais diferenças entre as condições, as diferenças foram sondadas com teste t pareado entre cada par de condições (com o p valor corrigido com o método Bonferroni-Holm). Essa abordagem baseada em múltiplas comparações representa uma análise de alta sensibilidade com intuito de verificar possíveis alterações de potência nas regiões centromediais e controlaterais associadas às condições. 
Por fim, foi testada a correlação de eventuais diferenças nas potências de alpha e beta entre as condições com os scores dos questionários.

\section{RESULTADOS}

Inicialmente, no estudo, buscou-se identificar a correlação da empatia com a ansiedade social. Através da aplicação da Escala de Liebowitz ${ }^{26}$, usada para avaliar ansiedade social, e da Escala Multidimensional de Reatividade de Davis (EMRI) ${ }^{27}$ em 70 voluntários, foi feita a seguinte tabela, usando como referência a Escala de Liebowitz.

A correlação (Pearson) entre os escores globais das escalas de empatia e ansiedade social foi signitificativa entre essses 70 voluntários $(p=0.0151)$. O Gráfico 1 mostra esta comparação.

Tabela 1. Relação dos escores obtidos nas subescalas de "Medo/ansiedade" e "Evitação" da escala de Liebowitz em 70 voluntários. Os escores são apresentados em ordem decrescente

\begin{tabular}{|c|c|c|c|c|c|c|}
\hline \multirow[t]{2}{*}{ Voluntário } & \multirow[t]{2}{*}{ Idade } & \multirow[t]{2}{*}{ Sexo } & \multirow[t]{2}{*}{ EMRI } & \multicolumn{3}{|c|}{ Liebowitz } \\
\hline & & & & Medo/ansiedade & Esquiva & Total \\
\hline 1 & 21 & F & 73 & 54 & 54 & 108 \\
\hline 2 & 22 & $\mathrm{~F}$ & 81 & 47 & 47 & 94 \\
\hline 3 & 20 & M & 77 & 54 & 40 & 94 \\
\hline 4 & 26 & M & 69 & 45 & 49 & 94 \\
\hline 5 & 18 & $\mathrm{~F}$ & 64 & 45 & 47 & 92 \\
\hline 6 & 21 & $\mathrm{~F}$ & 76 & 39 & 46 & 85 \\
\hline 7 & 19 & $\mathrm{~F}$ & 79 & 43 & 38 & 81 \\
\hline 8 & 20 & $\mathrm{~F}$ & 65 & 46 & 34 & 80 \\
\hline 9 & 22 & M & 98 & 37 & 38 & 75 \\
\hline 10 & 20 & $\mathrm{~F}$ & 73 & 31 & 44 & 75 \\
\hline 11 & 22 & $\mathrm{~F}$ & 58 & 38 & 36 & 74 \\
\hline 12 & 21 & M & 79 & 40 & 33 & 73 \\
\hline 13 & 21 & $\mathrm{~F}$ & 70 & 36 & 36 & 72 \\
\hline 14 & 20 & $\mathrm{~F}$ & 52 & 27 & 41 & 68 \\
\hline 15 & 19 & M & 56 & 34 & 33 & 67 \\
\hline 16 & 21 & $\mathrm{~F}$ & 73 & 30 & 35 & 65 \\
\hline 17 & 20 & M & 72 & 23 & 42 & 65 \\
\hline 18 & 20 & M & 72 & 41 & 22 & 63 \\
\hline 19 & 20 & M & 56 & 41 & 22 & 63 \\
\hline 20 & 20 & M & 65 & 17 & 45 & 62 \\
\hline 21 & 20 & $\mathrm{M}$ & 64 & 33 & 29 & 62 \\
\hline 22 & 20 & $\mathrm{~F}$ & 71 & 29 & 32 & 61 \\
\hline 23 & 20 & M & 68 & 30 & 31 & 61 \\
\hline 24 & 19 & $\mathrm{~F}$ & 76 & 30 & 29 & 59 \\
\hline 25 & 19 & $\mathrm{M}$ & 72 & 28 & 31 & 59 \\
\hline 26 & 22 & $\mathrm{~F}$ & 79 & 33 & 25 & 58 \\
\hline 27 & 19 & $\mathrm{M}$ & 73 & 32 & 26 & 58 \\
\hline 28 & 18 & $\mathrm{M}$ & 71 & 25 & 33 & 58 \\
\hline 29 & 24 & $\mathrm{M}$ & 60 & 26 & 31 & 57 \\
\hline 30 & 19 & $\mathrm{~F}$ & 78 & 32 & 23 & 55 \\
\hline 31 & 20 & $\mathrm{M}$ & 80 & 27 & 26 & 53 \\
\hline 32 & 19 & $\mathrm{M}$ & 74 & 28 & 25 & 53 \\
\hline 33 & 22 & $\mathrm{M}$ & 58 & 21 & 32 & 53 \\
\hline 34 & 19 & $\mathrm{~F}$ & 70 & 23 & 29 & 52 \\
\hline 35 & 24 & F & 88 & 24 & 26 & 50 \\
\hline 36 & 22 & F & 78 & 27 & 22 & 49 \\
\hline 37 & 18 & $\mathrm{~F}$ & 68 & 21 & 27 & 48 \\
\hline 38 & 22 & M & 60 & 28 & 19 & 47 \\
\hline 39 & 23 & $\mathrm{M}$ & 64 & 27 & 18 & 45 \\
\hline 40 & 20 & $\mathrm{~F}$ & 69 & 31 & 13 & 44 \\
\hline 41 & 20 & M & 72 & 20 & 22 & 42 \\
\hline
\end{tabular}


Mello AAF, Ramos RT. Supressão de ritmo mu durante exposição a estímulos associados a ansiedade social

Tabela 1. Relação dos escores obtidos nas subescalas de "Medo/ansiedade" e "Evitação" da escala de Liebowitz em 70 voluntários. Os escores são apresentados em ordem decrescente continuação

\begin{tabular}{|c|c|c|c|c|c|c|}
\hline \multirow[t]{2}{*}{ Voluntário } & \multirow[t]{2}{*}{ Idade } & \multirow[t]{2}{*}{ Sexo } & \multirow[t]{2}{*}{ EMRI } & \multicolumn{3}{|c|}{ Liebowitz } \\
\hline & & & & Medo/ansiedade & Esquiva & Total \\
\hline 44 & 19 & M & 74 & 19 & 21 & 40 \\
\hline 43 & 20 & M & 66 & 29 & 13 & 42 \\
\hline 44 & 19 & M & 74 & 19 & 21 & 40 \\
\hline 45 & 19 & M & 69 & 17 & 22 & 39 \\
\hline 46 & 18 & M & 75 & 21 & 17 & 38 \\
\hline 47 & 20 & $\mathrm{~F}$ & 58 & 16 & 22 & 38 \\
\hline 48 & 27 & M & 59 & 17 & 20 & 37 \\
\hline 49 & 21 & $\mathrm{~F}$ & 58 & 22 & 15 & 37 \\
\hline 50 & 19 & $\mathrm{~F}$ & 75 & 17 & 18 & 35 \\
\hline 51 & 20 & M & 69 & 14 & 21 & 35 \\
\hline 52 & 21 & $\mathrm{~F}$ & 67 & 18 & 17 & 35 \\
\hline 53 & 22 & M & 68 & 18 & 16 & 34 \\
\hline 54 & 20 & M & 65 & 19 & 15 & 34 \\
\hline 55 & 19 & M & 57 & 19 & 15 & 34 \\
\hline 56 & 20 & M & 66 & 18 & 15 & 33 \\
\hline 57 & 20 & $\mathrm{~F}$ & 70 & 6 & 26 & 32 \\
\hline 58 & 19 & $\mathrm{M}$ & 66 & 8 & 21 & 29 \\
\hline 59 & 19 & $\mathrm{~F}$ & 58 & 19 & 10 & 29 \\
\hline 60 & 19 & $\mathrm{M}$ & 55 & 2 & 27 & 29 \\
\hline 61 & 18 & $\mathrm{~F}$ & 54 & 15 & 13 & 28 \\
\hline 62 & 19 & $\mathrm{~F}$ & 64 & 7 & 20 & 27 \\
\hline 63 & 21 & $\mathrm{~F}$ & 64 & 13 & 13 & 26 \\
\hline 64 & 18 & $\mathrm{~F}$ & 60 & 13 & 12 & 25 \\
\hline 65 & 22 & $\mathrm{M}$ & 52 & 17 & 8 & 25 \\
\hline 66 & 22 & $\mathrm{~F}$ & 81 & 11 & 13 & 24 \\
\hline 67 & 19 & $\mathrm{M}$ & 70 & 12 & 9 & 21 \\
\hline 68 & 20 & $\mathrm{~F}$ & 68 & 11 & 6 & 17 \\
\hline 69 & 22 & $\mathrm{M}$ & 70 & 9 & 7 & 16 \\
\hline 70 & 21 & $\mathrm{M}$ & 71 & 7 & 3 & 10 \\
\hline
\end{tabular}

Gráfico 1: Teste de correlação de Pearson para as escalas de Ansiedade Social e Empatia para 70 voluntários

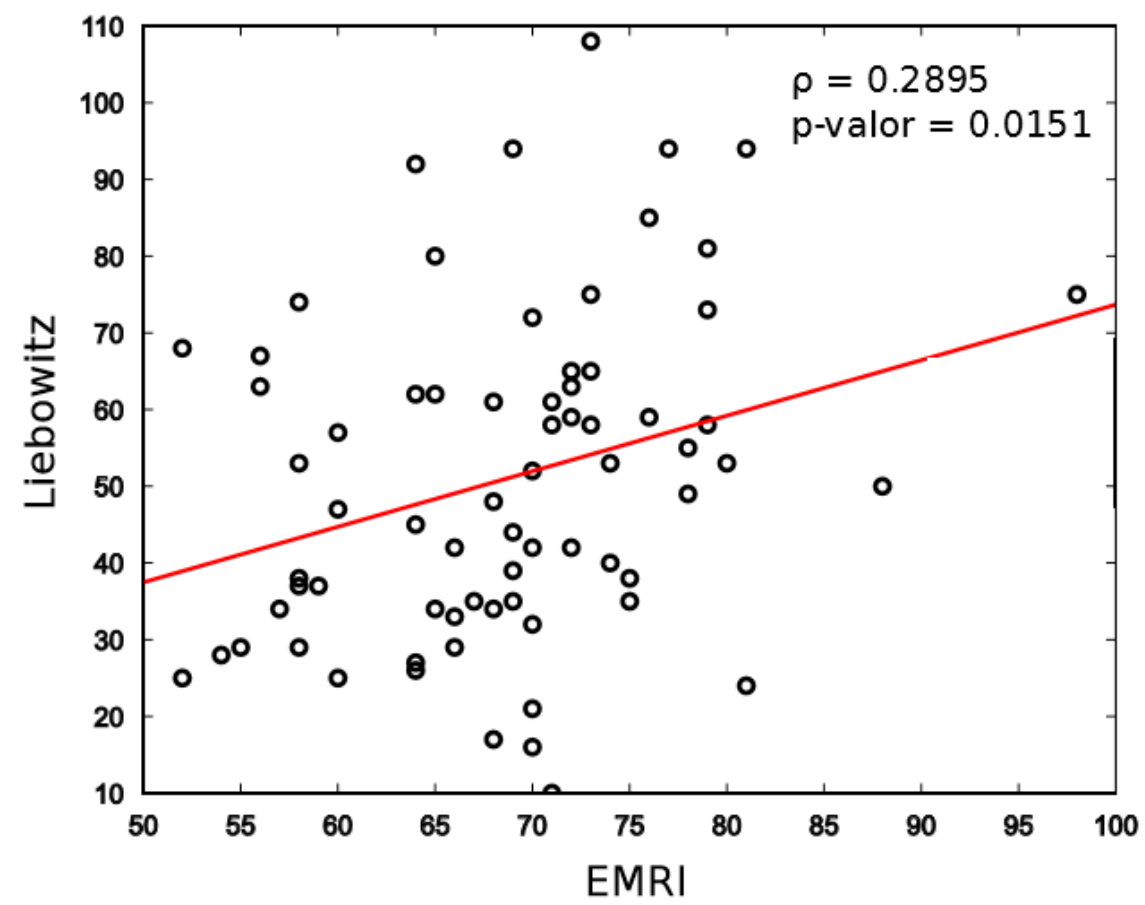


Na sequência, recrutaram-se para a próxima etapa do estudo 16 voluntários com escores variados, entre eles indivíduos com escores altos na escala de ansiedade social (voluntários 1, 4, 6, 7, 8) e indivíduos com escores mais baixos (voluntários 67 e 69). A amostra foi complementada com os voluntários 14, 17, 20, 21, 25, 29, 38, 41 e 43.

Ao comparar novamente os resultados das escalas, apenas com os voluntários que passaram pela fase do EEG (os 16 citados acima), para avaliar se a correlação entre os escores das escalas se mantinha, não foi encontrada a mesma significância. Portanto, não foi observada nesse estudo a relação direta entre esses dois parâmetros, a ansiedade social e a empatia.

$\mathrm{Na}$ etapa da realização do EEG, tinha-se como objetivo identificar a atividade dos neurônios espelho, a partir da análise da supressão de ritmo mu (um tipo de ritmo alpha somato-motor) em áreas frontoparietais. Inicialmente, os dados foram analisados por meio da técnica de Análise de Componentes Independentes ${ }^{28}$, em que foi possível retirar artefatos do EEG e sinais cerebrais não desejados no estudo, porém com esse método poucos componentes foram identificados como correspondentes ao ritmo mu.

Em uma nova análise, com o cálculo do espectro de potência de Fourier com método de Welch (janelamento de $1024 \mathrm{~ms})^{29}$, identificou-se potência de alpha com a frequência na banda de 8 a $14 \mathrm{~Hz}$, porém não em região frontoparietal, que poderia indicar um padrão de ritmo mu, e sim em região occipital. A análise post hoc foi feita a partir dos resultados obtidos em região parieto-occipital com a seleção de 10 eletrodos que apresentaram efeito principal de condição (Figura 1, superior).

A comparação post hoc revelou, portanto, a presença de atividade alpha em região occipital. Além disso, por meio da análise exploratória do registro do EEG, mais especificamente do achado de atividade alpha, nas três condições (primeira pessoa, terceira pessoa e terceira pessoa + movimento), evidenciou-se uma diferença significativa entre os padrões de atividade neuronal associados a primeira e terceira pessoa $(p=0.007$, Figura 1 , inferior).

\section{DISCUSSÃO}

Este estudo não encontrou uma correlação significativa entre medidas de empatia e ansiedade social e a intensidade de inibição do ritmo mu em regiões frontoparietais, como previsto. A avaliação deste achado deve ser considerada em função de algumas questões percebidas ao longo da execução do projeto.

Primeiro, observou-se que a correlação entre os escores das escalas de empatia e ansiedade social evidenciada na amostra geral de 70 voluntários não foi reproduzida no subgrupo de 16 voluntários convocados para os testes em laboratório. Esta observação sugere um grau de heterogeneidade da amostra selecionada maior que o esperado, o que pode dificultar que um efeito sutil como

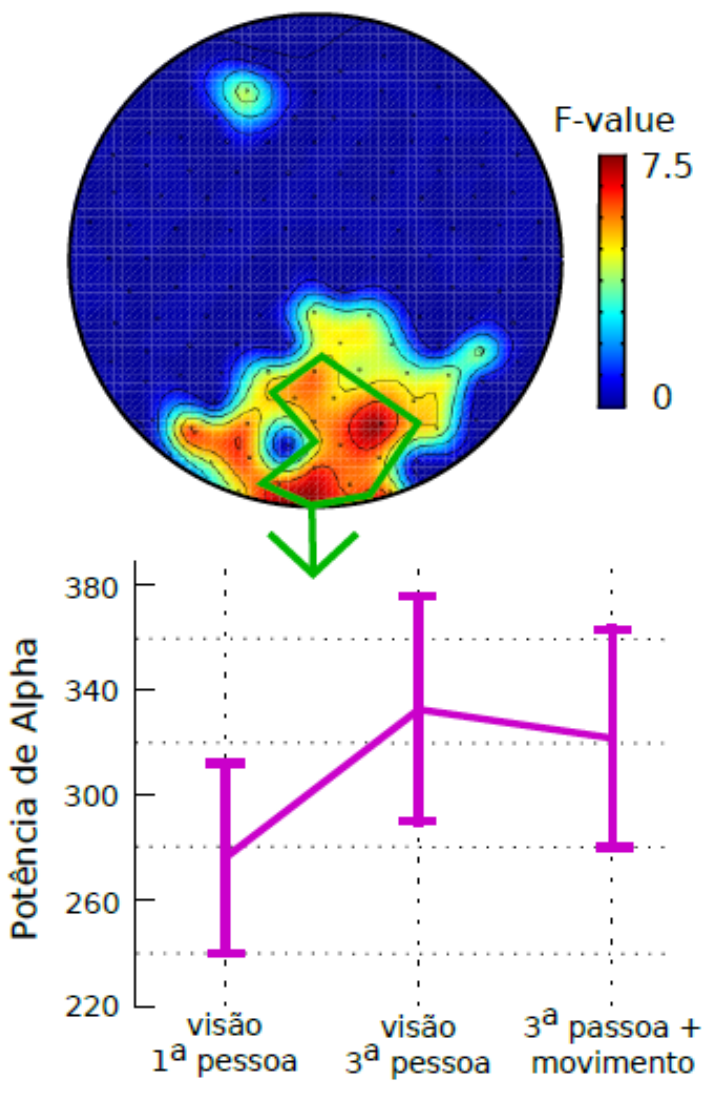

Figura 1. Padrão de atividade (banda de 8 a $14 \mathrm{~Hz}$ ) nas três condições de avaliação

a supressão de certos padrões de atividade cortical seja observado. Um cuidado maior na seleção de participantes e na dupla verificação dos escores de escalas daqueles indivíduos selecionados para testes deve ser observado em próximos experimentos.

Segundo, a maior potência de atividade na banda alpha foi observada em regiões occipitais e não em regiões frontoparietais, como o esperado. Esta diferença quanto à localização prevista dos eventos sugere que a atividade perceptiva visual induzida pelos estímulos utilizados possa ter sido mais intensa do que o esperado. Este fenômeno poderia, em tese, reduzir a relação sinal-ruído dos sinais detectados e reduzir a probabilidade de detecção de outros padrões de atividade cortical.

Embora os resultados obtidos não corroborem a hipótese de uma correlação entre a atividade de neurônios espelho e a gênese da ansiedade social, eles revelaram uma diferença significativa entre as condições de estímulo em primeira versus em terceira pessoa em regiões occipitais. Este achado certamente merece mais atenção em futuros estudos, pois parece estar associado à forma pela qual uma mesma situação é apresentada ao observador.

Em resumo, estes resultados parciais sugerem que o estudo dos padrões de atividade elétrica cortical 
permite abordar o efeito do contexto de observação sobre o processamento de informações cognitivosociais. O aperfeiçoamento deste modelo pode levar

\section{REFERÊNCIAS}

1. Cattaneo L, Rizzolatti G. The mirror neuron system. Arch Neurol. 2009;66(5):557-60. doi: 10.1001/archneurol.2009.41.

2. Pineda JA. The functional significance of mu rhythms: translating "seeing" and "hearing" into "doing". Brain Res Brain Res Rev. 2005;50(1):57-68. doi: 10.1016/j. brainresrev.2005.04.005

3. Makeig S, Westerfield M, Jung T-P, Enghoff S, Townsend J, Courchesne E, Sejnowski TJ. Dynamic brain sources of visual evoked responses. Science. 2002;295(5555):690-4. Erratum in: Science. 2002;295(5559):1466. doi: 10.1126/ science.1066168.

4. Oberman LM, Pineda JA, Ramachandran VS. The human mirror neuron system: a link between action observation and social skills. Soc Cogn Affect Neurosci. 2007;2(1):62-6. doi: $10.1093 / \mathrm{scan} / \mathrm{ns} 1022$.

5. Trilla Gros I, Panasiti MS, Chakrabarti B. The plasticity of the mirror system: how reward learning modulates cortical motor simulation of others. Neuropsychologia. 2015;70:255-62. doi: 10.1016/j.neuropsychologia.2015.02.033.

6. van Leeuwen WS, Wieneke G, Spoelstra P, Versteeg H. Lack of bilateral coherence of mu rhythm. Electroencephalogr Clin Neurophysiol. 1978;44(2):140-6. doi: 10.1016/00134694(78)90260-2.

7. Pfurtscheller G, Brunner, C, Schlögl, Lopes Da Silva A, FH. Mu rhythm (de)synchronization and EEG single-trial classification of different motor imagery tasks. Neuroimage. 2006;31(1):153-9. doi: 10.1016/j.neuroimage.2005.12.003.

8. Duntley SP, Kim AH, Silbergeld DL, Miller JW. Characterization of the mu rhythm during rapid eye movement sleep. Clin Neurophysiol. 2001;112(3):528-31. doi: 10.1016/ S1388-2457(00)00559-9.

9. Pineda JA, Allison BZ, Vankov A. The effects of selfmovement, observation, and imagination on mu rhythms and readiness potentials (RP's): toward a braincomputer interface (BCI). IEEE Trans Rehabil Eng. 2000;8(2):219-22. doi: $10.1109 / 86.847822$.

10. Babiloni C, Carducci F, Cincotti F, Rossini PM, Neuper C, Pfurtscheller G, Babiloni F. Human movement-related potentials vs desynchronization of EEG alpha rhythm: a highresolution EEG study. Neuroimage. 1999;10(6):658-65. doi: 10.1006/nimg.1999.0504.

11. Buccino G, Binkofski F, Fink GR, Fadiga L, Fogassi L, Gallese V, Seitz RJ, Zilles K, Rizzolatti G, Freund H-J. Action observation activates premotor and parietal areas in a somatotopic manner: an fMRI study. Eur J Neurosci. 2001;13(2):400-4. doi: 10.1111/j.1460-9568.2001.01385.x.

12. Rizzolatti G, Fogassi L, Gallese V. Neurophysiological mechanisms underlying the understanding and imitation of action. Nat Rev Neurosci. 2001;2(9):661-70. doi: $10.1038 / 35090060$.

13. Leslie KR, Johnson-Frey SH, Grafton ST. Functional imaging of face and hand imitation: towards a motor theory ao desenvolvimento de novas abordagens no estudo da fisiopatologia da ansiedade em geral e da ansiedade social em particular.

of empathy. Neuroimage. 2004;21(2):601-7. doi: 10.1016/j. neuroimage.2003.09.038.

14. Stroganova TA, Orekhova EV, Posikera IN. EEG alpha rhythm in infants. Clin Neurophysiol. 1999;110(6):997-1012. doi: 10.1016/S1388-2457(98)00009-1.

15. Blanchard DC, Griebel G, Pobbe R, Blanchard RJ. Risk assessment as an evolved threat detection and analysis process. Neurosci Biobehav Rev. 2011;35(4):991-8. doi: 10.1016/j.neubiorev.2010.10.016.

16. Singer T, Seymour B, O’Doherty J, Kaube H, Dolan RJ, Frith CD. Empathy for pain involves the affective but not sensory components of pain. Science. 2004;303(5661):1157-62. doi: 10.1126/science. 1093535 .

17. Singer T, Frith C. The painful side of empathy. Nat Neurosci. 2005;8(7):845-6. doi: 10.1038/nn0705-845.

18. Shamay-Tsoory S, Harari H, Szepsenwol O, Levkovitz Y. Neuropsychological evidence of impaired cognitive empathy in euthymic bipolar disorder. J Neuropsychiatry Clin Neurosci. 2009;21(1):59-67. doi: 10.1176/appi.neuropsych.21.1.59.

19. Brown EC, Gonzalez-Liencres C, Tas C, Brüne M. Reward modulates the mirror neuron system in schizophrenia: A study into the mu rhythm suppression, empathy, and mental state attribution. Soc Neurosci. 2016;11(2):175-86. doi: 10.1080/17470919.2015.1053982.

20. Lepage JF, Lortie M, Deal CL, Théoret H. Empathy, autistic traits, and motor resonance in adults with Turner syndrome. Soc Neurosci. 2014;9(6):601-9. doi: 10.1080/17470919.2014.944317.

21. Baird AD, Scheffer IE, Wilson SJ. Mirror neuron system involvement in empathy: a critical look at the evidence. Soc Neurosci. 2011;6(4):327-35. doi: 10.1080/17470919.2010.547085.

22. Ferrari PF. The neuroscience of social relations. A comparativebased approach to empathy and to the capacity of evaluating others' action value. Behaviour. 2014;151(2-3):297-313. doi: 10.1163/1568539X-00003152

23. Iacoboni M. Imitation, empathy, and mirror neurons. Annu Rev Psychol. 2009;60:653-70. doi: 10.1146/annurev. psych.60.110707.163604.

24. Pineda JA, Hecht E. Mirroring and mu rhythm involvement in social cognition: are there dissociable subcomponents of theory of mind? Biol Psychol. 2009;80(3):306-14. doi: 10.1016/j.biopsycho.2008.11.003.

25. Pineda JA, Friedrich EV, LaMarca K. Neurorehabilitation of social dysfunctions: a model-based neurofeedback approach for low and high-functioning autism. Front Neuroeng. 2014;7:29. doi: 10.3389/fneng.2014.00029.

26. Santos LF. Estudo da validade e fidedignidade da Escala de Ansiedade Social de Liebowitz - versão auto-aplicada [dissertação]. Ribeirão Preto: Departamento de Psiquiatria, Faculdade de Medicina de Ribeirão Preto, Universidade de São Paulo; 2012. 
Mello AAF, Ramos RT. Supressão de ritmo mu durante exposição a estímulos associados a ansiedade social

27. Koller SH, Camino C, Ribeiro J. Adaptação e validação interna de duas escalas de empatia para uso no Brasil. Estud Psicol (Campinas). 2001;18(3):43-53. doi: 10.1590/S0103$166 \times 2001000300004$.

28. Simas E. Introdução à análise de componentes independentes e suas aplicações. In: III Semana de Engenharia Elétrica da UFBA; 2008. p.7-10.

29. Moore A, Gorodnitsky I, Pineda J. EEG mu component responses to viewing emotional faces. Behav Brain Res. 2012;226(1):309-16. doi: 10.1016/j.bbr.2011.07.048.

Artigo recebido em: 29.03.17

Artigo aceito em: 29.06.17 